antecedent(s), and no special arc between the variable and the consequent(s). Both representations have nodes representing the propositions of the antecedent(s) and the consequent(s). Looked at in this way, Fahlman's restriction that "No clause may be part of the specification of more than one *EVERYnode" [p. 161] can be seen as eliminating the possibility of representing statements of the form (Ax)(Ay) $\{S(x, y)->I(x, y)\}$.

Before concluding, I must make one comment on a passage which I fear illustrates a common attitude among AI researchers. Fahlman points out that, "If an object is said to exist within an area, that means that it exists somewhere within the area; if a statement is said to be valid within an area, it means that it is valid everywhere within the area" [p. 114, italics in the original]. He then comments that "This difference in the behavior of existence and statement-scoping came as something of a surprise to me -- I am told that the logicians have known something of this sort all along, but they tend not to express it in these terms. This difference caused a lot of trouble until I understood what was happening" [p. 115]. The first point is that this is a nice statement of the reason AI researchers should study logic. Perhaps, if he had, Fahlman would have been saved a lot of trouble. The other point is that if a researcher discovers that he has rediscovered a distinction or issue already discussed by others in different terms, he should rewrite his own discussion in the existing terms, giving the appropriate credit and providing the connections to his readers, not dismiss the older work in a couple of sentences and contribute to the proliferation of jargon. The issue is no less than the respectability of AI.

What makes these complaints more serious is that this book represents a good piece of work, already having an impact on the "knowledge representation" community. It is certainly must reading for anyone also involved in the inheritance hierarchy brand of representation.

Stuart C. Shapiro, SUNY-Buffalo

\section{Understanding Spoken Language}

Donald E. Walker, Editor

Elsevier North-Holland, New York, 1978,

420 pp., Paperback, \$9.95, ISBN 0-444-00287-1.

In 1970 the Advanced Research Projects Agency decided to fund six research projects aimed at developing systems that were capable of understanding connected speech. From 1971 to 1975 , this research was carried out. This book is a collection of articles (most of which have been published separately) which grew out of the final report of the speech understanding group at SRI International. Despite its title, its stated purpose is to describe SRI's speech understanding system rather than speech understanding in general, and it contains much material pertinent to understanding written language as well as speech.

Although the introductory and concluding material attempts to unify the book, it remains a collection of very separate articles rather than a unified whole. As a consequence it suffers from the common problems of books of this type: inadequate cross referencing, poor transitions between chapters, and no index. The absence of an index is a serious problem that is compounded by the fact that the table of contents contains only three levels of structure; more detailed outlines of the contents are found at the beginning of each chapter. Fortunately the references have been merged into a single list. The reference list is very good, in part because it is not too long to scan easily.

The signal processing part of speech understanding is given the barest mention because SRI did not do work in that area; the book concentrates on the higher level aspects of the understanding process.

The first chapter is a nicely written introduction and overview by Donald Walker. It describes the organization of the ARPA speech understanding effort and outlines the SRI system. The second chapter, by William Paxton, quickly plunges the reader into a rather detailed description of the language definition system which was used to define the language that the system would understand. These definitions were then compiled into a form that the executive system, which controlled the other components of the system, would understand. The language definition consisted of a lexicon (words and "multiwords" with grammatical categories, grammatical features, and associated semantic information) and composition rules (phrase structure rules augmented by procedures to be executed whenever the rule constructs a phrase). The procedures gave values to attributes of the phrase as a function of the attributes of its constituents and judged the acceptability of the phrase on a number of grounds such as acoustic properties, syntactic properties (such as mood and number), semantic properties (using the semantic network representation discussed further on), and discourse information to handle anaphora and ellipsis. Much of the complexity of the language definition derives from the fact that it must screen out bad input rather than just recognize good input as many grammars do.

In discussing the executive system itself, much space is devoted to historical background, comparisons with other speech understanding systems, and the experimentation (using analysis of variance) that was 
done to determine the best control strategy to use. The control issues that were tested were left to right processing through the sentence versus an "island driven" strategy, examining all the words that might be present in a given location at once versus taking them one at a time, doing time-consuming but accurate context checking as soon as possible versus delaying such checking, and focusing the processing on a single hypothesis versus skipping around to whatever hypothesis seemed best at the time. The average reader will probably be more interested in the results of the experiments than the details of them, however.

The third chapter explains the semantic component of the system, which was represented by the partitioned network scheme of Gary Hendrix. With many detailed examples, Hendrix shows how the partitioning scheme was used to encode quantifiers and logical connectives (conjunction, disjunction, negation, implication), to form associations between semantic objects and the syntactic units of the input, to distinguish between new and old information, to encode multiple hypotheses, to allow sharing of representations among competing hypotheses, and to define hierarchies for discourse analysis. The semantic component of the system used this formalism to filter out combinations of words that were acoustically and syntactically acceptable but semantically unacceptable; it also constructed a representation of the meaning of good interpretations for other components to use, and could make predictions of words or structures that were likely to occur in other parts of the utterance.

The next chapter is devoted to Barbara Grosz's work on discourse knowledge. After showing examples of how the focus of a dialogue affects the identification of definite noun phrases, word sense interpretation, pronominal reference, and ellipsis, she discusses (again, in more detail than some readers would wish) the analysis of actual problem solving and question answering dialogs which were examined to provide the basis for a representation of focus that would enable the SRI system to use focus in its semantic interpretations. The notion of focus spaces which was derived from these experiments was represented in Hendrix's partitioned network formalism and used for resolution of noun phrases, inferencing, reference resolution, and other high level aspects of sentential processing. The problems of shifting focus, reinvoking an old focus of attention, and dealing with ellipsis are also covered in detail.

The fifth chapter comprises three sections relating to the problem of responding to an utterance once it has been understood. This is unfortunately limited in scope, since the major emphasis of the project was on understanding rather than responding to spoken language. Gary Hendrix writes on the problem of interacting with the deduction component and an English generator to formulate a reply. Richard Fikes and
Gary Hendrix detail the deduction component, and Jonathan Slocum's section deals with generating an English description of a semantic structure. The conclusion, written by Ann Robinson, summarizes the work and points out issues relating to other areas of research.

One of the chief features of the book is the large number of illustrations and detailed examples, including as an appendix a short but well chosen example of the entire processing of a single utterance.

Someone already familiar with the ARPA speech project will gain little from this book, and someone interested in a general overview of the speech understanding problem and the ARPA project's results would do better to look elsewhere [1, 2]. What this book has to offer is something rare but not unimportant in the literature: a detailed description of a single large and complex system. One can get from it not only an understanding of how that system worked but also an excellent understanding of the important pieces of that work which have had a continuing influence in the field of computational linguistics since the termination of this particular project.

Madeleine Bates, Bolt Beranek and Newman

\section{References}

[1] Klatt, Dennis. Review of the ARPA Speech Understanding Project. J. Acoust. Soc. Am. 62, 1977

[2] Lea, Wayne A. (Ed.). Trends in Speech Recognition. PrenticeHall, Englewood Cliffs, N.J., 1980.

The Process of Question Answering A Computer Simulation of Cognition

Wendy G. Lehnert

Lawrence Erlbaum Associates, Hillsdale, N.J., 1978, 278 pp., \$16.50, ISBN 0-470-26485-3.

This book can be reviewed from two perspectives: (1) as a general and technical introduction to the complexities and subtleties regarding human understanding of and response to questions and (2) as a much more comprehensive system, intended to produce not only humanlike interpretations of questions, but doing so in ways that model human thought processes. Lehnert appears to have both approaches in mind, emphasizing the second more, but it is with respect to only the first that the book is successful.

From this first perspective, then, there is no other single source that so completely and persuasively illustrates the many-faceted problems of translating a person's question (e.g., "Do you have a match?" vs. "Do you have a hangover?") into a valid interpretation of the questioner's desires or information requirements (e.g., "Give me a light" vs. "How is your physical state after last night?"). Lehnert provides abundant evidence for the necessity of utilizing a wide 